\title{
Geospatial images in the acquisition of spatial knowledge for wayfinding
}

\author{
Pyry Kettunen ${ }^{1}$, Katja Irvankoski ${ }^{2}$, Christina M. Krause ${ }^{2}$, \\ Tapani Sarjakoski ${ }^{1}$, and L. Tiina Sarjakoski ${ }^{1}$ \\ ${ }^{1}$ Department of Geoinformatics and Cartography, Finnish Geodetic Institute, Masala, Finland \\ ${ }^{2}$ Cognitive Science, Institute of Behavioural Sciences, University of Helsinki, Helsinki, Finland
}

Received: March 19,2012; returned: June 6, 2012; revised: August 31, 2012; accepted: September 8, 2012.

\begin{abstract}
Geospatial images, such as maps and aerial photographs, are important sources of spatial knowledge that people use for wayfinding. The rapid development of geodata acquisition and digital graphics has recently led to rather complete geographic coverage of both traditional and novel types of geospatial images. Divergent types of geospatial images vary in their support of human acquisition of spatial knowledge. However, evaluative studies about the acquisition of spatial knowledge from the diversity of geospatial images have been rare. In this article, we review a variety of literature about the acquisition of spatial knowledge while paying particular attention to the role of geospatial images. Based on the literature, we present a framework of image parameters that characterize the acquisition of spatial knowledge from geospatial images: vantage point, number of visible vertical features, and visual realism. With the help of the framework, we evaluate commonly used geospatial images. In concordance with the previous experiments, our evaluation shows that the different types of geospatial images have large differences in the types of spatial knowledge they support and to what extent. However, further experimentation is needed in order to better understand the human cognitive needs for geospatial images and to develop more useful geospatial images for wayfinding.
\end{abstract}

Keywords: geospatial image, spatial knowledge, wayfinding, cognitive map, landmark, route, vertical, realism 


\section{Introduction}

Wayfinding is an everyday activity that people perform during the simplest transitions inside buildings as well as during complex route traversals through urban environments. Wayfinding requires complex cognitive processing and allocates a significant portion of the human cerebral system. The vast majority of wayfinding is carried out unconsciously, using highly developed internal spatial representations of familiar environments, such as walking from an office to a lunch restaurant. However, cognitively challenging wayfinding occurs in unfamiliar environments, where new environments must be integrated into the internal spatial representation.

Learning unfamiliar environments often begins with surveying a geospatial image such as a map or an aerial photograph. The supply of such images has exploded during the last few decades due to the development of geospatial data capture and visualization techniques in parallel with the available computing power and communication technologies. Consequently, there are various types of geospatial images available with significant geographic coverages. For example, accurate satellite images are easily available from all around the world.

Although geospatial images have been intensively developed, research into their effects on human thinking has only followed gradually. Human wayfinding has been an active field of research for the psychological and cognitive sciences for a century, but controlled experimentation on the use of geospatial images has been carried out only during last few decades (for example, early experiments by Thorndyke and Hayes-Roth [69| and Passini [58|). Research in psychology has traditionally applied maps merely as one kind of visual stimuli without a thorough view of the semantics involved and how they affect map reading and interpretation. On the other hand, research in cartography has not comprehensively considered results of cognitive science [45|. Due to the advent of advanced geovisualization during last ten years, there exist many kinds of commonly used geospatial images that are imperfectly studied with regards to human cognition, such as navigator and street views.

This article presents an analytical review on geospatial images in light of theories on the acquisition of spatial knowledge for wayfinding. The aim of the review is to clarify linkages between the cognitive and geographic information sciences in order to elaborate analyzing geospatial images in wayfinding context, which can later help to develop the usability of geospatial images. The review is based on both theoretical and experimental literature on wayfinding and geovisualization (Sections 2 and 31. Leaning on the literature, a framework is created in order to evaluate the support of geospatial images for wayfinding (Section 4). Eventually, common types of geospatial images are evaluated with the help of the framework created and needs for further experimental research on these image types are addressed (Section 51. In the end of the article, a summary is given of the review, and implications and restrictions of the review are discussed (Section 6).

\section{Spatial knowledge and wayfinding}

Spatial knowledge is information and understanding about the physical environments. Human spatial knowledge is tightly linked to, and often even defined by, the action of finding and following routes from one place to another [41]. These everyday route activities be-

www.josis.org 
came named wayfinding by the 1980s in spatial cognition research [58|. The widely accepted definition of wayfinding is that of Golledge [27 p. 6]: "Wayfinding is the process of determining and following a path or route between an origin and a destination." Wayfinding contributes mainly to local and regional spatial knowledge because the vast majority of human wayfinding is conducted at these scales. Global scale spatial knowledge, in contrast, is almost always acquired from geospatial images such as maps or satellite images. Our interest here is restricted to spatial knowledge at the large-scale spaces of everyday pedestrian wayfinding such as walking in a city or hiking in nature. We consider large-scale spaces as defined by Kuipers [41 p. 129]: spaces "whose structure cannot be observed from a single viewpoint."

\subsection{Internal spatial representation and cognitive map}

Research into spatial knowledge spans decades and multiple scientific disciplines, including psychological learning theory, cognitive science, neuroscience, and geography $|3|$. Initial studies of the human internal storage of spatial information emerged about a century ago. In the then newborn discipline of psychology, Trowbridge [71| was one of the first to report about what he called imaginary maps. Trowbridge considered imaginary maps as false knowledge of spatial reality and discussed reasons that caused people to hold such erroneous representations in their minds. The research field of spatial cognition as we see it today developed through behavioral experiments on animals, which culminated on Tolman's [70| conception of cognitive map, which he showed to exist in rats with maze navigation experiments. Tolman [70| assumed cognitive maps being a characteristic of humans as well as rats and so launched the research field of cognitive mapping.

In the 1970s, cognitive mapping research had developed a reasonably well-established view on the acquisition and storage of human spatial knowledge. In their overview paper on the nature of cognitive maps, Downs and Stea [18 p. 8] define cognitive mapping as "a process composed of a series of psychological transformations by which an individual acquires, codes, stores, recalls, and decodes information about the relative locations and attributes of phenomena in his everyday spatial environment." The product of this process is a cognitive map upon which spatial decisions and strategies are based [18|. The notion of cognitive map has been rewritten many times since then. Presently, cognitive maps are widely conceptualized as internal representations emphasizing spatial relations of places and objects (e.g., [27|). It should be noted that although spatial mental representations are called cognitive maps, they are systematically distorted, fragmented, and multimodal rather than coherent map-like representations containing only information acquired through visual modality [72].

The amount, accuracy, and variety of the acquired spatial knowledge (or cognitive map) of a particular environment can be evaluated through various measures. Images of previously encountered scenes or landmarks of an environment can be used to study the recognition memory for places $\mid 14$ 50|. Knowledge of spatial relations between landmarks and other objects may be inferred through the accuracy of localization, distance, and direction estimations between the subject and objects or between objects $[23,57,66,69 \mid$. In addition to these measures, knowledge of spatial layout can be evaluated by identifying the validity or accuracy and variety of the sketch maps, which subjects draw from their memory $[9.14]$. The above measures cover the spatial layout of the environment as only a two-dimensional plane, but also the elevation or height of an object can be estimated |64|. Finally, the appli- 
cability and accuracy of the acquired spatial knowledge can be measured through actual wayfinding behavior; that is, the completion time, number of steps, or wrong turns during travel from the starting point to the destination [23.50 73|.

\subsection{Elements of wayfinding}

Wayfinders have to be able to identify features in the environment and recognize places and scenes in order to orient themselves and successfully navigate without getting lost [37.58]. Place recognition can be based on previous experience or on wayfinding aids, such as maps or verbal instructions. Scenes or places may be recognized by feature cues-that is, distinct landmarks or groups of landmarks_-or by geometric cues, such as the configuration of buildings or the shape of a road grid [13|. Landmarks are important in wayfinding, especially around decision points, such as cross-roads, demonstrated in several experiments (e.g., [12.36 53|). For landmarks to be of use, they should be relatively stable, easily detectable, and recognizable in the environment $[26,68 \mid$.

During wayfinding, knowledge of one's own location has to be updated constantly in a process called spatial updating $[40 \mid$. Updating may be based on the changing of relative positions of the landmarks or on path integration process, that is, dead reckoning. In dead reckoning, updating is based on recent movement of the body and on optical flow, which does not entail the recognition of landmarks [26.53|. However, the path integration process is insufficient for navigation over longer distances due to the accumulation of error [53|.

While moving in an environment, one may either relate the information about objects and places in the environment to oneself as an egocentric representation; or to the other objects in the environment or to a coordinate system as an allocentric representation [4 53]. Egocentric representation may be understood as egocentric coordinates where distances and directions are related to the navigating person as a body-centered vector [40], or as sensorimotor contingencies. Sensorimotor contingencies relate actions to resulting perceptual change, for example, how visual input changes while moving |51|. Recent research into representational perspectives suggests that allocentric and egocentric views exist in parallel and it is a matter of personal preference or task requirements which is applied [11 51|. The existence of these two frames of reference is supported by the research finding that different areas of the brain have a distinguishable role in tasks demanding the application of egocentric or allocentric frames of reference. Further evidence can be found in the translation between allocentric to egocentric frames of reference $[4 \mid$.

\subsection{Goals and conditions of wayfinding}

The goal of the wayfinding is usually some other activity than the wayfinding itself [34]. Wayfinding tasks can be divided into three different types according to the goals of wayfinding. These three task types are traveling to a familiar destination, exploratory travel in the environment, and traveling to a novel destination |2|. The goals and tasks affect wayfinding so that the granularity of a mental route model depends on the needs of the ongoing activity: which landmarks or objects are included and how detailed the internal representations of the landmarks are [34|.

Several environmental and activity-related conditions affect human wayfinding. With regards to environmental conditions, different types of terrain activate spatial cognitive processes differently. For example, urban environments require different kinds of land-

www.josis.org 
mark recognition than do natural environments [59.63|. Seasonal changes are shown to affect the way people perceive the environment [63|. Daylight certainly has a considerable effect on the human wayfinding processes. In addition, wayfinding in a familiar environment is less demanding than wayfinding in a novel environment. When traveling to a novel destination, a person needs some kind of a navigational aid such as a map, verbal description, or written instructions [2]. Considering activity-related conditions, the locomotion modality of the wayfinder affects the perception of space and construction of spatial knowledge [38|.

The goals and conditions of wayfinding must be considered when investigating the support of geospatial images because different types of images are likely to vary in the support they provide for spatial cognition in different conditions.

\section{Acquisition of spatial knowledge}

Human spatial knowledge may be acquired either from direct experience or from external spatial representations, such as geospatial images or verbal descriptions. In this section, we review research that discusses the fundamental processes of spatial knowledge acquisition from direct experience. We also consider external spatial representations by reviewing research on geospatial images, on which we focus later in this article.

\subsection{Spatial knowledge acquisition from direct experience}

According to the established and commonly used model of Siegel and White [65], spatial knowledge is stored in three knowledge types, which together constitute the internal spatial representation. In their model, each knowledge type or level supports the formation of the next more structured level.

Landmark knowledge concerns "unique configurations of perceptual events" |65. p. 23]that is, landmarks. Landmark knowledge serves the identification of known places and is the first type of spatial knowledge that a child becomes capable of acquiring. It is also the first type of knowledge which adults learn in an unfamiliar environment. Landmark knowledge is mainly visual for humans and is based on visual recognition memory [65|.

Route knowledge builds on "sensorimotor routines for which one has expectations about landmarks and other decision points" [65. p. 24]. In other words, route knowledge is sequential knowledge of landmarks and connecting paths. Route knowledge of an unfamiliar environment is formed in the human mind on the basis of landmark knowledge by ordering landmarks according to their sequence of encountering on a route. Due to route knowledge, people are able to perform basic wayfinding: transitions from one place to another.

Configuration knowledge is the most structured type of spatial knowledge "that gives its owner an advantage in way-finding and organizing experience" [65 p. 24]. Configuration knowledge has geographic features or objects organized in relation to each other so that the understanding of environmental patterns and planning of new routes is possible. Siegel and White [65| argued that configuration knowledge is a highly integrated form of a reservoir of route knowledge. Configuration knowledge only develops with repeated experience in an originally unfamiliar environment once one has enough route knowledge 
that can be interrelated. Configuration knowledge is often called survey knowledge and its conception is parallel to that of a cognitive map.

Reflecting on the spatial learning literature, Siegel and White [65| present processes through which the three types of spatial knowledge are stored in the human mind and articulate how these types pile on top of each other, eventually ending up with an accurate and functional internal spatial representation of the environmental setting. They stress the importance of learning landmarks as the base of all spatial knowledge and propose humans possess a specialized memory for this, which they call "recognition-in-context" memory.

Later research on landmarks has verified the importance of landmark knowledge for spatial knowledge. For example, Passini [58| shows that landmark recognition is a dominant process in wayfinding. Participants in his experiments expressed poor spatial knowledge of a shopping mall in their route- and configuration-like sketch maps, but were still able to successfully find their way in the mall. Passini concludes that the participants were able to recognize landmarks in the context of navigation even if they were not able to recall them when drawing sketch maps.

The spatial knowledge acquisition model of Siegel and White [65| concentrates on learning unfamiliar environments through direct experience, in which locomotion plays a central role. However, even if the direct experience probably serves a wayfinder in shaping the most complete state of spatial knowledge possible with all the visual landmark details available (Lynch [46| mentions even details of door handles as landmarks in his analysis of Boston), spatial knowledge of an unknown area is often acquired first by external spatial representations either in verbal or visual forms, such as verbal descriptions or maps. External spatial representations often provide better views on route and configuration types of spatial knowledge than on landmark knowledge, so that route and configuration knowledge are acquired before landmark knowledge, which is not considered in the model of Siegel and White $|65|$.

Indeed, Siegel and White's model has been criticized for a too inflexible view with regards to the order of learning and for too large a focus on primary landmark knowledge [6.35,52|. Montello [52 | criticized, above all, the learning of configuration knowledge only after other knowledge types. Montello [52| proposed an alternative framework that allows gradual metric learning from the beginning of the exposure to an unknown environment. Experimental evidence has supported this so-called "continuous framework" |10.35|. In this article, we apply Siegel and White's [65| types of spatial knowledge with geospatial images, but consider Montello's [52| continuous framework for the development of the knowledge types. This means that acquiring knowledge does not entail direct experience of the environment but, rather, the knowledge can be acquired also from external geospatial representations or from general understanding about the arrangement of elements or landmark features in the environment.

\subsection{Spatial knowledge acquisition from geospatial images}

In this article, the term geospatial images is used to refer to external visuo-spatial representations of spatial knowledge, in contrast to internal visuo-spatial representations discussed in Section 2.1. Geospatial images provide spatial knowledge of large environments without direct experience in the environment. This knowledge can be integrated into the viewers' cognitive map and be used in spatial reasoning and wayfinding.

Www.josis.org 
Previous spatial knowledge research shows that using geospatial images activates the same regions of the brain as direct experience in the environment (e.g., [1 44 49]). The same brain structures process spatial information regardless of the origin of spatial information. Consequently, with respect to spatial knowledge acquisition, geospatial images can be studied based on the theories of spatial cognition, which have been developed through experimentation in real environments.

Systematic psychological studies on spatial knowledge acquisition from geospatial images are much more recent than the studies investigating the acquisition from direct experience (among the first were [58 69|, see [65| for review dating back to 19th century). Different kinds of geospatial images have existed since humankind began to draw, but heavy development of geospatial images has emerged along with the digital visualization technologies only in the last few decades. Psychological research into geospatial images has grown in parallel with the development of geovisualization. Thorndyke and Hayes-Roth $|69|$ compared spatial knowledge acquired from navigation and maps with a hypothesis that the resulting cognitive maps differ. They used quantitative measures of distance and angle between distant points in order to settle the accuracy of cognitive maps, which the subjects developed in the experiment. The results showed that the subjects estimated the angles and route distances more accurately with direct navigation experience, and Euclidean distances more accurately with map viewing. In terms of spatial knowledge types, this indicates that navigation produces principally route knowledge and map viewing configuration knowledge. Thorndyke and Hayes-Roth's $|69|$ experiment also showed that navigating participants improved their spatial knowledge over time, resulting in the same level of configurational knowledge as the map learners. In contrast, repeated exposure to maps did not enhance either route or configuration knowledge.

Richardson et al. [62] conducted a comparable study to Thorndyke and Hayes-Roth's [69| using navigation, maps, and virtual 3D environment for the acquisition of spatial knowledge. They replicated the result of the more accurate Euclidean distance estimates when studying maps compared to direct experience. However, unlike Thorndyke and Hayes-Roth $|69|$, they controlled the study for map alignment and found a strong effect on direction and route distance estimates: these measures were estimated significantly more accurately if the headings of the participant and map were aligned. When the headings were misaligned, no differences were found in the direction and route distance estimations between viewing maps and navigating in real environment. This result accords with those from other alignment studies (e.g., [42 75|). In addition to map alignment control, Richardson et al. [62| also controlled the amounts of exposure to maps and navigation to be equal in their experiment, which was not the case with the study of Thorndyke and Hayes-Roth [69|. This can partially explain the accuracy differences in the route knowledge measures. Virtual 3D environment provided the weakest support for spatial knowledge acquisition in the study of Richardson et al. [62|. Specifically, learning directions and configuration knowledge was weak in the virtual environment. Richardson et al. [62| suggested this to result from difficulty in updating heading after a rotation in the virtual environment possibly due to an insufficient optic flow.

Münzer et al. [54| investigated the effect of the visual presentation mode, visuo-spatial working memory capacity, and gender on wayfinding performance as well as its effect on route and configurational learning through two experiments. In the first experiment, an egocentric eye-level view was compared with two types of allocentric views: a traditional 2D map showing buildings and streets and a compass view showing only destinations on 
the map but no buildings or street layout information. The hypothesis was that the egocentric view would support the wayfinding performance and route knowledge but would lead to impaired configurational learning compared to other two displays. Whereas the egocentric view showed only one part of the route at a time, the other two views covered the whole experiment area with the starting point and the destination in the same view. Therefore, the second hypothesis was that these visualization modes would support configurational learning while impairing wayfinding performance. The effect of a map view and a compass view on configurational knowledge was assessed with sketch maps and the accuracy of direction estimates between the visited destinations. In the second experiment, the authors compared the effect of user-aligned visual presentation to a display where north was always at the top of the display. Both of the views were allocentric aerial views showing only part of the route and surrounding environment at decision points (not the whole route with all the destinations). The hypothesis was that the automatic user alignment would lead to weaker configurational knowledge than the north-on-top display. In the latter, it was expected that the user would be better aware of how the different parts of the experiment area would be related to each other, due to the stable frame of reference during the route execution. In addition, the user-aligned view was expected to produce fewer wayfinding errors than the north-on-top view.

The results of the first experiment of Münzer et al. [54] showed that the visualization mode would support either wayfinding performance or configurational learning. Wayfinding performance was weaker with the two configurational modes while these modes supported configurational learning. Wayfinding was supported with the visualization modes that presented only restricted regions around decision points with both the egocentric eyelevel and aerial vertical visualization modes. However, either one of these presentation modes did not enhance route knowledge compared to the configurational modes. The more detailed map mode did not affect the quality of sketch maps compared to the more simple compass mode, which did not produce more accurate direction estimates compared to the map presentation mode. In the second experiment the map alignment did not affect configurational learning or wayfinding performance. Taken together, the support for wayfinding seemed to be related to the size of the area shown at one time on the display rather than to the vantage point whereas better configurational learning required that the whole experimental area would be shown in a single view.

Furthermore, spatial knowledge acquisition from geospatial images is importantly affected by the goals of map reading [9|. The results of Brunyé and Taylor [9| showed that if the participants were instructed to learn routes between locations using a map, they answered more accurately to questions which considered egocentric perspective. Similarly, if the participants were instructed to learn the overall layout of the environment they answered more accurately to questions made from allocentric perspective. The goal of studying the map affected the frame of reference in which the map information was processed.

In the present paper, our major interest falls on geospatial images in the static form. However, the use of moving views in dynamic and interactive virtual environments affects considerably the spatial knowledge transfer. Effects are due to such visual cues as optic flow, which may help in estimating route distances and turning directions (route knowledge) based on the changes of the view [10 15, 62|. Dynamism in geospatial views may activate human spatial updating processes, which are not heavily used in the case of static images [40 74|. Interactivity in geospatial views is also known to influence spatial knowl- 
edge acquisition due to the activity of the viewer, but the effects may be positive or negative depending on the task at hand [15].

In the experiments investigating whether route learning is plain serial learning of sequential landmarks, Buchner and Jansen-Osmann [10| compared conditions where pictures of stuffed animals were shown to participants either in static or dynamic form. These conditions were further divided into scenes with a black background or a contextual background, which presented the objects in a segmented hallway with stonewalls; the length of the segments varied. The results of the serial recall test showed that participants remembered more landmarks in their correct positions in the dynamic hallway context. In the second experiment of the same study $|10|$ the authors replicated the same design except that the segments of the hallway in the dynamic hallway context were exactly the same length. In this second experiment the results of the serial recall test did not differ between the groups. These results indicate that both context and dynamism affect learning of the position of a landmark in a sequence through providing metric information about the relative position of the landmarks. In turn, this suggests that displays where the viewer can move around, such as street views, may provide better support for landmark memory compared to static displays.

\section{Evaluating wayfinding support of geospatial images}

Geospatial images are typically presented on planar surfaces, such as paper and screen. A transformation is needed in order to depict the physical, three-dimensional world on a two-dimensional surface. This transformation is defined by a set of choices on the visual representation and underlying geodata, which we call image parameters. The image parameters determine which aspects of the real world a geospatial image highlights. Image parameters may lead to divergent perceptions of a particular area. Thus, image parameters affect the spatial knowledge which can be acquired from geospatial images. In this article, we base our analysis of geospatial images of physical reality on three image parameters: vantage point, number of visible vertical features, and visual realism. In the following sections, these parameters are described and examined in relation to the types of spatial knowledge, which previous research has identified. The results of previous experimental studies are reviewed, which demonstrate the effects of each parameter on the degree of support of geospatial images for wayfinding.

The presented framework considers geospatial images as direct derivatives of visual reality, which is relevant for wayfinding in the real world. It allows for classifying the utility of geospatial images for wayfinding purposes without treating the semiotics of the contents of the images (for classification of spatial representations see [47|).

\subsection{Vantage point}

The vantage point determines the viewpoint of a geospatial image. From a cognitive perspective, the vantage point is essentially determined by the vertical viewing angle of an image, which defines how visible vertical features can appear in the image. With regards to spatial knowledge acquisition, three main cases can be identified for vantage points in geospatial images (Figure 1): 
Aerial vertical From an aerial vertical vantage point, the ground surface is represented directly from above with a constant scale throughout the image. Vertical faces of objects, such as building facades and cliffs, are visible only in the case of perspective projection, which causes them to be slightly visible away from the projection center.

Aerial oblique An aerial oblique vantage point represents the ground from an aerial viewpoint with a vertical viewing angle between the horizontal and vertical directions. The most convenient vertical viewing angle is around 45 degrees, which causes both horizontal and vertical features to be equally visible [7].

Ground horizontal A ground horizontal vantage point is a couple of meters above the ground and the vertical viewing angle is close to the horizontal direction (parallel to the ground), which causes dominance of vertical features in the view, such as facades of buildings.

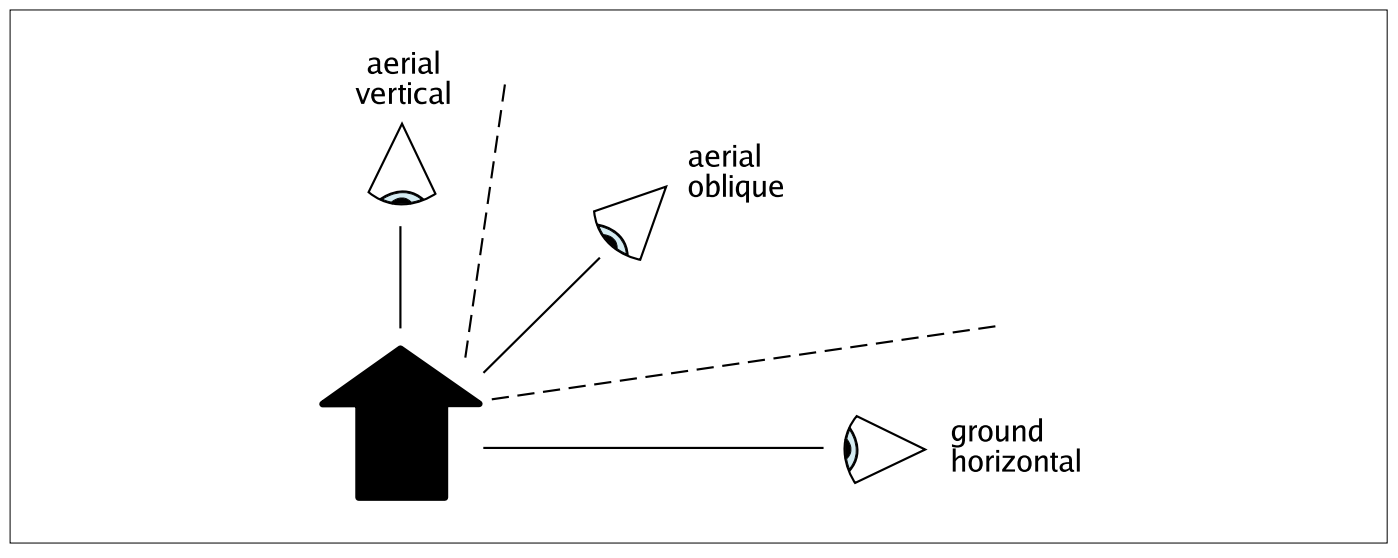

Figure 1: The three main cases for vantage points in geospatial images.

In addition to the main cases as such, vantage points can also be mixed in geospatial images; separate feature types may be depicted from different vantage points. For example, typical touristic city maps apply a vertical aerial vantage point for a street network and an oblique aerial vantage point for buildings and significant landforms.

The effect of vantage point on spatial knowledge has been rarely studied separately. User experiments of geospatial images often consider 3D views in a holistic way or as parts of virtual 3D environments which involve a moving view. Fontaine [23| did study the effect of vantage point in static geospatial images by investigating navigation time between two landmarks at an unfamiliar metro station where wayfinders acquired spatial knowledge from real navigation, floor plans (in our terms, aerial vertical vantage point), frontal views (a special case of ground horizontal vantage point), or axonometric representations (aerial oblique vantage point). Learning by navigation in the real environment outperformed learning from any of the geospatial images, which was explained by the good visibility through the metro station in reality [23|. Considering geospatial images, axonometric representation allowed the best wayfinding performance and supported specifically women's wayfinding. However, Euclidean distances were estimated more accurately using floor plans and frontal views. Fontaine [23| also tested for the accuracy of landmark localization and found that axonometric representation allowed for the best performance. 
Her results suggest that aerial oblique vantage point supports the acquisition of all types of spatial knowledge because it shows both the landmarks and the configuration of the environment, although distances may be more accurately observed from an aerial vertical vantage point.

Münzer et al. [54| found in their study of eye-level (ground horizontal vantage point) and 2D map (aerial vertical vantage point) route views that the two presentation modes did not differ in their support for wayfinding as long as the visible areas were similar in size. The result suggests that sufficient landmark knowledge for successful wayfinding can be acquired from the two vantage points. However, comprehensive 2D maps which covered the whole area of the experiment caused significantly lower wayfinding performance. The small scale and large number of landmark features in complex configurations may have made the aerial vertical vantage point too cognitively challenging for navigation decisions on-route. The study did not indicate differences in the acquired configurational knowledge between the different conditions.

Users often prefer aerial oblique vantage point to aerial vertical one, especially in environments where height dimension is significant such as mountains, city centers, or underground environments. Schuchardt and Bowman [64| reported on the effectiveness of aerial oblique vantage point in their experiment on levels of immersion in a virtual cave environment. The participants were cave experts who evaluated virtual reality environments highly useful: $91 \%$ would use the virtual environment in place of or in addition to 2D cave plans, which apply aerial vertical vantage point.

Many experiments have been conducted where dynamic virtual 3D environments are compared with other types of geospatial images, particularly traditional twodimensional maps [33, 57,60,66,73]. Virtual environments usually employ ground horizontal vantage point as they aim at cloning real-world visual experience.

Waller et al. [73| reported an experiment in which the participants walked repeatedly through a curtain maze blindfolded. Subjects relied on the spatial knowledge they had acquired either from real navigation, a 2D map (aerial vertical vantage point), or a virtual environment (ground horizontal vantage point). The route following performance was best for the map learners, which indicates the utility of the aerial vertical vantage point. However, virtual environment learners who were given a long time for practicing, caught up the performance of map learners along with repetitions of the task. The experiment of Waller et al. [73| mainly measured the route and configuration knowledge because the blindfolded participants could not rely on the visual landmark knowledge in the navigation task.

The superiority of aerial vertical vantage point over ground horizontal vantage point for acquiring spatial knowledge for wayfinding has been observed also in the experiments of Oulasvirta et al. [57| and Hile et al. [33|. Both experimented using 2D and 3D views in mobile phones as navigation support for urban wayfinding. The study of Oulasvirta et al. [57| indicated that self-location is more efficient using 2D maps than $3 \mathrm{D}$ environment (aerial oblique and ground horizontal vantage points) because 2D maps provide direct configuration knowledge to orient oneself in the environment. A virtual 3D environment caused major usability problems due to the large amount of interaction needed to browse the environment. The preference for an aerial oblique vantage point was noted with $3 \mathrm{D}$ use, as users often tended to change the perspective into the bird's eye view. In the experiment of Hile et al. [33| the users had possibilities to employ both 2D orthophotos (aerial vertical vantage point) and street photos (ground horizontal vantage point) in a mobile phone aided route following task. They preferred 2D orthophotos most of the time, due to its view 
of the configuration of the route, but regularly changed to street photos at decision points in order to get confirming landmark knowledge for performing turns.

Two more supporting experiments on the acquisition of configuration knowledge from aerial vertical viewpoint are those of Sjölinder et al. [66| and Piovesana et al. [60|. Sjölinder et al. [66| found that a 2D map added confidence for navigation in a virtual 3D environment of a store. However, the 2D map did not affect navigation performance, which the authors explained by all the cognitive resources having been used for navigation only; there were no resources left for interpreting the map. Piovesana et al. [60| experimented on the use of Google Street View with or without a tangible tabletop 2D map navigation interface. The users of the tabletop 2D map interface turned out to learn the environment faster than those using Google Street View only.

In summary, aerial oblique vantage point has been shown to be the most advantageous one for acquiring spatial knowledge for wayfinding in the environments with considerable amount of vertical features $|23,64|$. Aerial oblique vantage point provides the viewer with both landmarks and configuration of the environment, both of which are needed for effective wayfinding. However, further experimentation is needed to understand more deeply the effects of an aerial oblique viewpoint on the spatial knowledge in different kinds of environments. In flat environment experiments considering such areas as cities or interiors of buildings, an aerial vertical viewpoint has constantly outperformed ground horizontal viewpoint due to its ease of use and interpretation. Nevertheless, repeated use of geospatial images that use ground horizontal vantage point may end up with comparable spatial knowledge to that acquired from the aerial vertical viewpoint.

\subsection{Number of visible vertical features}

In our framework, the number of visible vertical features refers to the quantity of perceptible vertical faces of objects in a geospatial image. The number of visible vertical features indicates how extensively surfaces with a vertical dimension are visualized in an image. This depends both on the vantage point of the geospatial image and on the available data on the vertical features.

The number of visible vertical features is highly significant for the spatial information processing of the viewer: people are used to conceptualize viewing based on the heights of spatial features-our everyday viewing scenes are dominated by vertical features as we are mostly watching to horizontal directions. In the case of geospatial images, the number of visible vertical features may vary continuously between non-existent and comprehensive (Figure 2). In some applications (typically navigators) only a certain portion of vertical features are drawn visible in order to highlight the respective landmarks.

Experimental results on the effect of the number of visible vertical features on spatial knowledge can be extracted, to a great extent, from the same studies as the results on the effects of vantage point (see Section 4.1). The studies on 3D views have shown the importance of the number of visible vertical features for acquiring landmark knowledge, which is crucial for wayfinding [33, 57, 62]. Among the geospatial images that Fontaine [23| used in her experiment, vertical features were visible in the frontal views and axonometric presentation. Both were proved to have a positive impact on the wayfinding performance at the metro station when compared to the floor plan, which contained no visible vertical features. Cavers in Schuchardt and Bowman's [64| study evaluated virtual 3D environments of cave systems as especially useful because they could perceive the elevations in the caves.

www.josis.org 


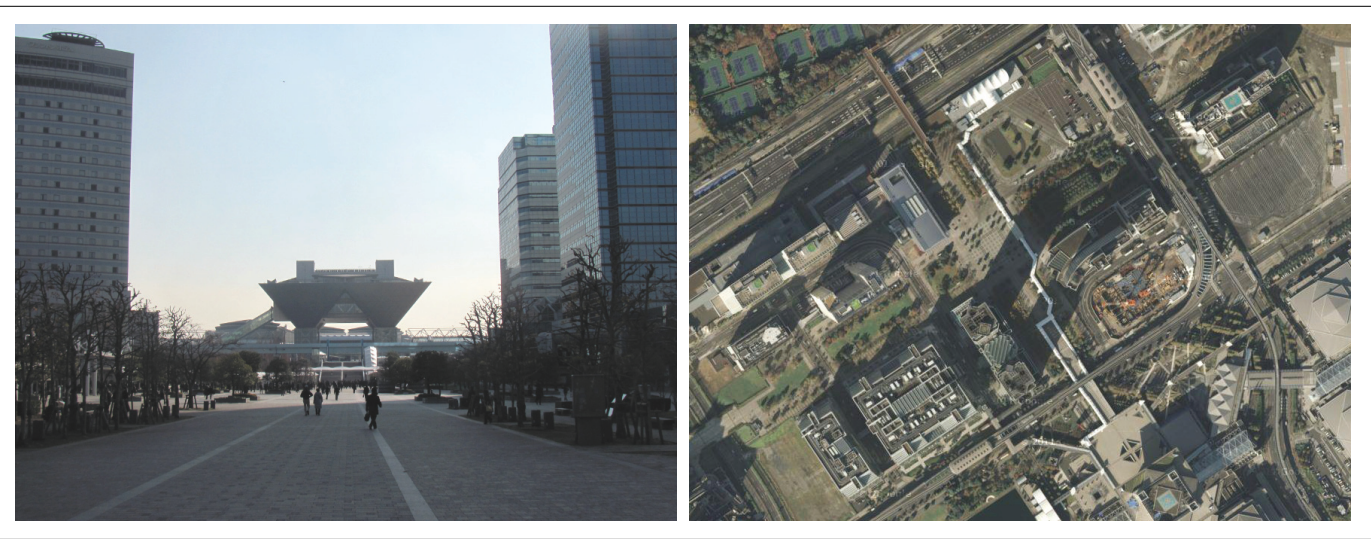

Figure 2: Number of visible vertical features is high in a horizontal photo (left) and low in a satellite image (right, same region (C) 2012 Google, Digital Earth Technology, DigitalGlobe, GeoEye, The Geoinformation Group, ZENRIN) [28|.

In the case of cave systems the vertical information probably accounts most importantly for the configuration knowledge of elevation, which is hard to extract from direct navigation experience or 2D cave maps.

The studies of Fontaine [23| and Schuchardt and Bowman [64| support the importance of the number of visible vertical features for spatial knowledge for wayfinding when viewed from the aerial oblique vantage point. Viewing from the ground horizontal vantage point alone does not create such a benefit because landmarks cannot be instantly associated together [57. 73|. However, the comprehensive number of visible vertical features from ground horizontal vantage point becomes more beneficial if integrated with the configuration knowledge provided by the aerial vertical vantage point $[6066]$.

\subsection{Visual realism}

Visual realism means the level of visual resemblance of a geospatial image with the real world. It defines the degree of abstraction [48| in a geospatial image and significantly affects the cognitive processing of the viewer. Low visual realism requires interpretation of the abstract symbology in the image but concentrates on essential features [5]. In contrast, high visual realism is easy to interpret but demands extraction of the relevant features. The visual realism in geospatial images may vary continuously between abstract and photorealistic (Figure 31, and mixed cases are also common.

The experimentation on visual realism has been most active in virtual 3D environments, which offer an adequate platform for changing the degree of realism and investigating its effect on spatial cognition [14|. Meijer et al. [50| present a significant positive effect of the degree of visual realism on the wayfinding performance in a virtual 3D environment of a supermarket. The users who used a high-realism environment for learning were faster and more accurate in all the spatial knowledge tasks than those who used a low-realism environment. The spatial knowledge tasks were route reversal, map identification, route drawing, and viewpoint recognition. However, the learning phase in the high-realism condition took a considerably longer time as the participants viewed the details of the high-realism 


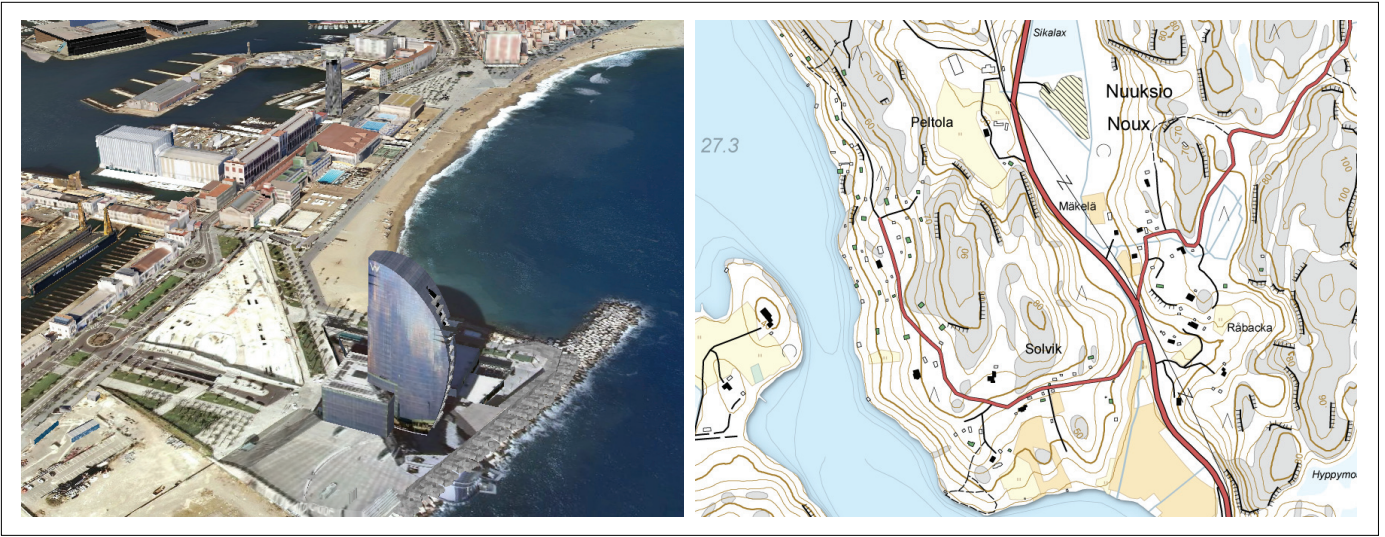

Figure 3: Visual realism is high in a photogrammetric 3D rendering (left (c) 2011 Google and Institut Cartogràfic de Catalunya) [29| and low in a topographic map (right) [56].

3D environment carefully. Further research should consider if the results are repeated with restricted learning times for both conditions.

Nikolic [55| studied the effects of screen size, stereoscopy, and visual realism on the acquisition of spatial knowledge and the formation of the cognitive map. She used a virtual 3D environment of a two-storey residence building. She noted that high realism of a virtual $3 \mathrm{D}$ environment did not enhance the cognitive map in all situations. In contrast, the use of large screen and stereoscopy in concert with a high degree of visual realism weakened the level of spatial organization in her experiments compared to a low level of realism. She explained the decrease of performance in configuration knowledge tasks by the participants focusing on details not necessary for building the cognitive map.

The effect of realism is often measured by the viewers' sense of presence: that is, how much the viewers feel as being truly in the virtual environment. It can be hypothesized that the higher the sense of presence, the more the spatial cognitive processes in a virtual environment resemble those in the real world. High sense of presence has been shown to correlate with high visual realism of a 3D environment in many studies (e.g., [8.67|) although not always [77|. Zimmons and Panter [77| suggested that the similar measures of presence for all levels of visual realism in their experiment resulted from the impressiveness of their task with a strong feel of danger. Having a natural feeling in a virtual environment probably enhances the spatial awareness and acquisition of spatial knowledge, especially in the case of landmark knowledge.

Users of virtual 3D environments tend to prefer high levels of realism. Cartwright [12] reports on users' wishes to have as much detail as possible in the virtual 3D environment of a city in which the users performed wayfinding tasks. The users asked for real-looking street signs, background images, traffic, and even electricity cables in the air. None of them reported that complex detail would have decreased their perception of the environment. However, Cartwright's [12] study also showed that high realism is not necessary throughout the model but it should be used particularly in the constituent places of wayfinding such as street corners. The latter observation is in line with the view of Döllner [17] who proposes non-photorealistic rendering to have high potential for creating effective geospa- 
tial images for the acquisition of spatial knowledge. The preference for a high level of realism has also been identified in situations where a more simplistic visualization has been more efficient. In these cases, the extra information has been of no use, or even disadvantageous, for the map task at hand [32| and this extra information has presented geographical complexity instead of or in addition to other information [31|. The reason for the users' preference for a high level of realism may be a higher confidence in the data quality in highly realistic geospatial images $|20,76|$. However, overconfidence on visual realism may lead to faster but more erroneous performance of orientation and direction estimation in tasks where a map has to be matched to scene or the other way around [16].

The effect of realism on spatial knowledge acquisition has been only partially studied in the case of photos. The convenience of aerial photos for acquiring spatial knowledge has been shown by Haynes et al. $|30|$. Their aerial photos were taken from an aerial oblique vantage point. The acquisition of spatial configuration knowledge was measured by a questionnaire, which concerned spatial characteristics of the viewed mountainous island with regards to volcanics. The aerial photos were found to be significantly better for the formation of a cognitive map than 2D maps or aerial oblique 3D maps. Still, further research is needed to understand how useful photos are for acquiring spatial knowledge. For example, experimental evidence is lacking about the spatial knowledge acquired from aerial orthophotos in comparison to 2D maps.

In summary, visual realism has been shown to affect the acquisition of spatial knowledge for wayfinding. The evidence from experiments in virtual 3D environments and on aerial oblique photos suggests that high visual realism is advantageous for gaining spatial knowledge. In addition, users prefer high realism in these environments even if more abstract representations would be more efficient for the task. However, evidence is lacking on the effects of visual realism in other geospatial images and on different types of spatial knowledge. Highly realistic virtual 3D environments probably support wayfinding and, especially, landmark knowledge in their resemblance to the real world. However, high realism in the form of photos might not be equally beneficial in the case of an aerial vertical vantage point and configuration knowledge, for example.

\section{Geospatial images frequently used for wayfinding}

There is a wide divergence among geospatial images. In this section, we review seven common types of geospatial images that, in our opinion, are frequently used. For each image type, there is a paragraph on the framework-based evaluation and a paragraph on experimental findings. For the framework-based evaluations, we discuss and evaluate the support of image types for the acquisition of different types of spatial knowledge based on the image parameters of our framework. The image parameters for the image types are collected in Table 1 and the evaluated support for spatial knowledge acquisition in Figure 4. For each image type, we further survey previous experimental findings.

Determining the values for image parameters may be ambiguous, particularly for the mixed cases. Here, we have chosen seven common types of geospatial images so that they favor an unambiguous determination of values. In this way, the image types here are ideal cases, which might not all exactly match real applications. Rather, these image types define a typology according to which geospatial images in real applications can be described and classified. 


\begin{tabular}{|c|c|c|c|}
\hline Geospatial image & Vantage point & $\begin{array}{l}\text { Number of visible } \\
\text { vertical features }\end{array}$ & Visual realism \\
\hline Symbolic 2D map & aerial vertical & non-existent & abstract \\
\hline Aerial orthophoto & aerial vertical & low & photorealistic \\
\hline Tilted 2D map & aerial oblique & non-existent & abstract \\
\hline Oblique aerial photo & aerial oblique & high & photorealistic \\
\hline Oblique 3D drawing & aerial oblique & high & low \\
\hline Panoramic street view & ground horizontal & comprehensive & photorealistic \\
\hline Ground-level 3D rendering & ground horizontal & comprehensive & high \\
\hline
\end{tabular}

Table 1: Image parameters in the reviewed types of geospatial images.

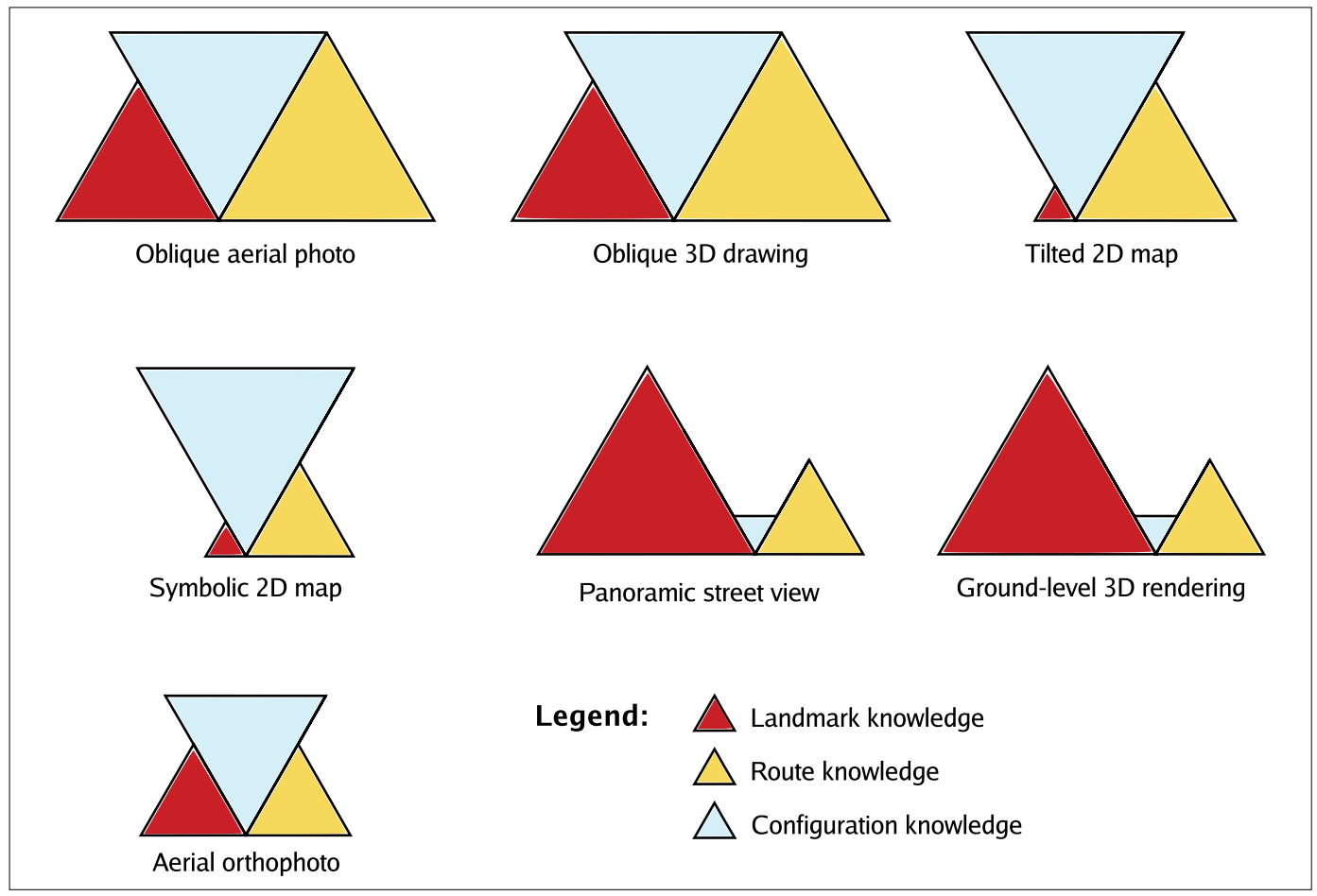

Figure 4: Framework-based evaluations of support of common geospatial images for the acquisition of spatial knowledge. The sizes of the triangles illustrate the evaluated support.

\subsection{Symbolic 2D map}

We call a symbolic 2D map (Figure 5) a traditional map that is drawn using symbolic geodata mapped on a projection plane using the normal projection: the map plane is fitted parallel with the ground surface using an appropriate map projection and viewed orthogonally from above. Symbolic 2D maps are the most common form of maps, and have been used for 
thousands of years. The idea behind this traditional map type lies in the human everyday conception of the world as a flat plane seen from above.

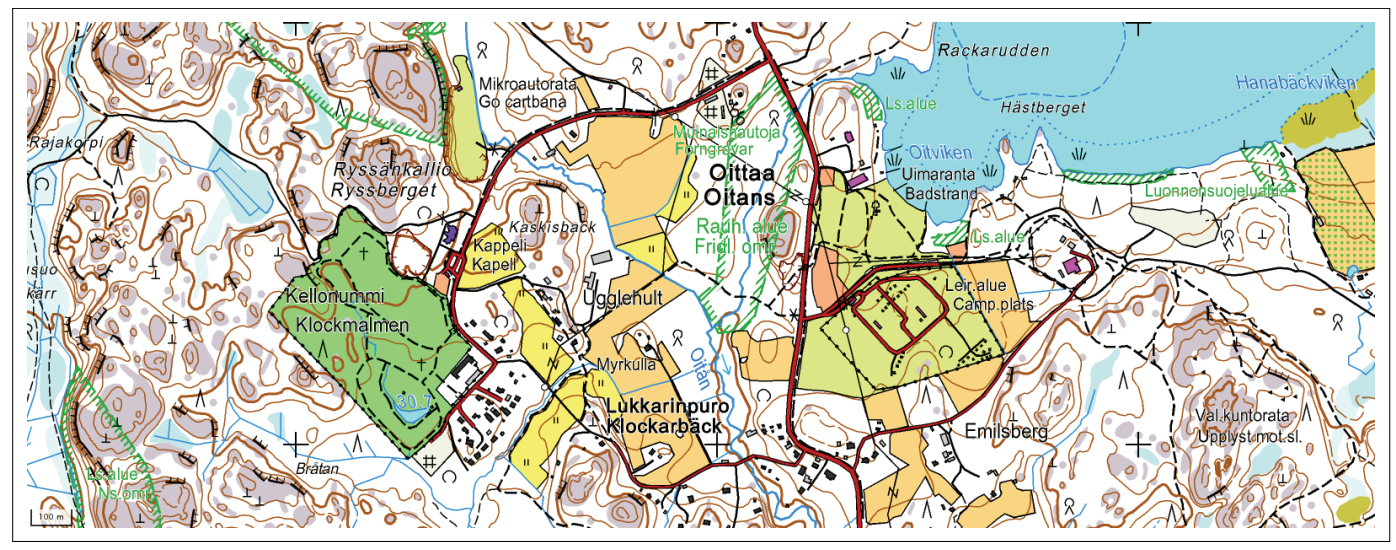

Figure 5: An example of symbolic 2D maps. Basic map (C)National Land Survey of Finland, permission no: 244/MML/12.

Framework-based evaluation In terms of our image parameters in this review, symbolic 2D maps apply the aerial vertical vantage point, provide no visible vertical features, and are abstract. Based on above analysis of image parameters (Section 4), symbolic 2D maps best support the acquisition of the configuration type of spatial knowledge as they make it easy to form a general understanding of an environmental setting due to the vantage point and no occluding vertical features. Route knowledge is also easily available, but landmark recognition knowledge as defined by Siegel and White [65| cannot be acquired as such.

Experimental findings Because symbolic 2D maps are the most produced and used type of geospatial images, a substantial body of user experiments has been conducted on them. Symbolic 2D maps have been explicitly rated efficient in rapidly providing spatial knowledge for wayfinding (e.g., [57 62.69.73|). The accuracy of the acquired knowledge is generally good and suitable to be used both for planning a route and conducting a route traversal. However, the utility of a symbolic 2D map depends on the skills of the viewer to interpret the map image. Symbolic 2D maps may burden viewers with high cognitive load [54 66], especially if they are not familiar with the cartographic symbols. For example, aligning a symbolic $2 \mathrm{D}$ map according to the heading of the viewer is an important task for the map use, the ease of which depends on the viewers' mental rotation abilities [42, 62] and their experience.

\subsection{Aerial orthophoto}

An aerial orthophoto (Figure 6) is a geospatial image composed of multiple aerial photos, which have been rectified to a specific scale and merged together to form a photo of a large area. Aerial orthophotos resemble symbolic 2D maps in viewing angle, which is orthogonal 
to the ground surface. The contents of aerial orthophotos are similar to what could be seen with the human eye from an aircraft.

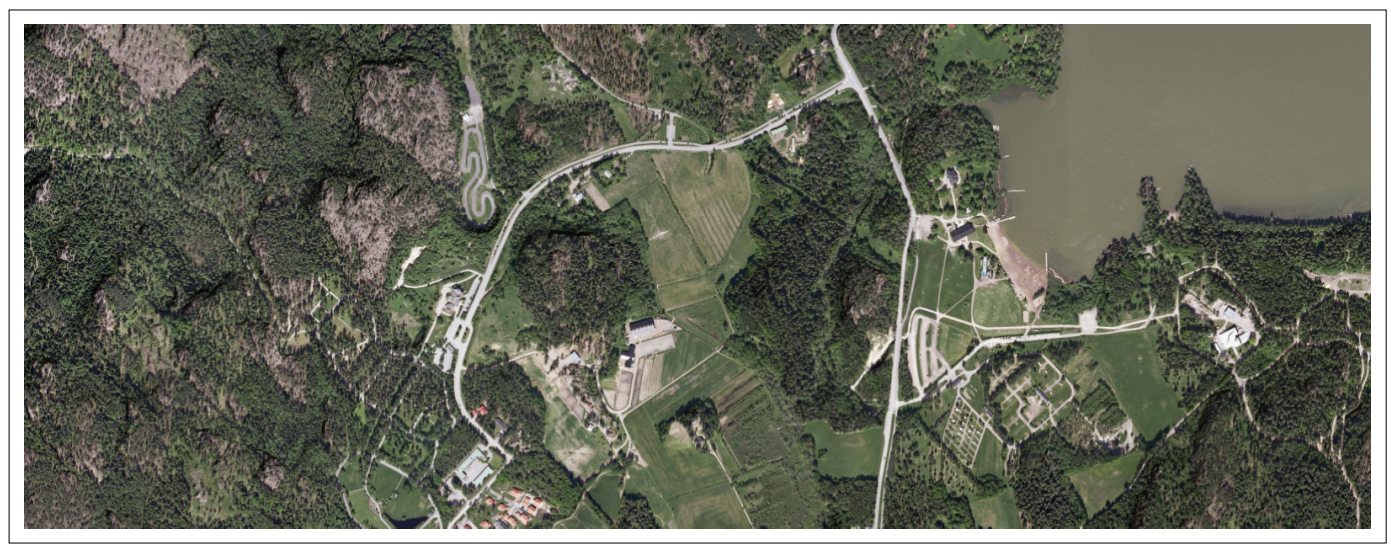

Figure 6: An example of aerial orthophotos.

Framework-based evaluation Aerial orthophotos apply the aerial vertical vantage point, contain low number of visible vertical features, and are photorealistic. As for symbolic 2D maps, aerial orthophotos best support the configuration type of spatial knowledge. However, due to the visual realism in aerial orthophotos, viewers are able to extract some landmark knowledge such as colors of the rooftops, types of trees, and so forth. On the other hand, the realism requires viewers to interpret the spatial structure in the image themselves. In addition, small-sized features cannot be discerned in small-scale aerial orthophotos even if they might have considerable importance for the spatial conceptualization of the surroundings. These aspects seem to make an aerial orthophoto a cognitively demanding spatial media. However, people are by experience readily able to interpret realistic views like orthophotos.

Experimental findings Experiments on the acquisition of spatial knowledge for wayfinding from pure aerial orthophotos are rare. Additional drawing contents have often been added on photos for experiments. Hile et al. [33| made their experiment on unaugmented aerial orthophotos and found that aerial orthophotos were advantageous for planning routes and for realizing the navigation. In order to corroborate the above analytical notions on the capabilities of spatial knowledge transfer through orthophotos, more controlled experiments are needed.

\subsection{Tilted 2D map}

We call a tilted 2D map (Figure 7) a type of geospatial images in which a symbolic 2D map is drawn in perspective projection. This image type has been common in navigating applications such as car navigators. 


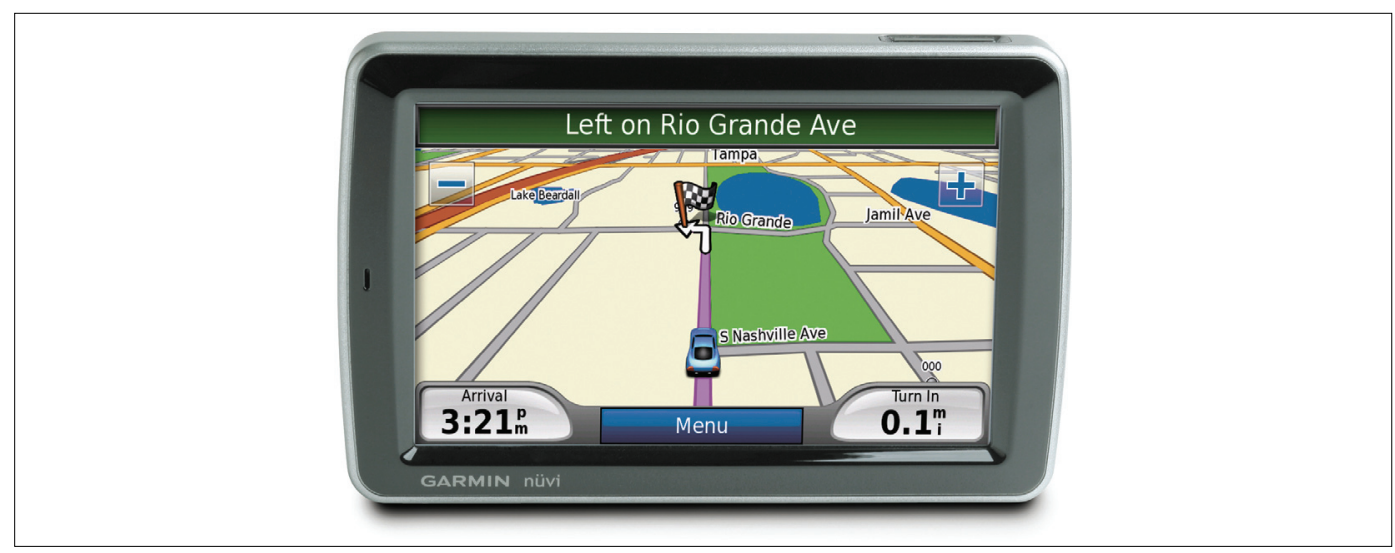

Figure 7: An example of tilted 2D maps (C)Garmin Ltd [25|.

Framework-based evaluation Tilted 2D maps apply the oblique aerial vantage point, contain no visible vertical features, and are visually non-realistic. The combination of the oblique aerial vantage point with perspective viewing projection and no visible vertical features creates a beneficial synthesis particularly for navigating: the diminishing scale in a view from the foreground to the background causes the nearby environment to be appear larger and, simultaneously, provides the viewer with a wide view of remote areas, assisting users in anticipating upcoming navigation actions. Also, vertical features do not occlude other features, which makes the contents of the view complete. In particular, this combination of image parameters supports the acquisition of the configuration type of spatial knowledge. Route knowledge is supported due to the egocentric vantage point. Landmark knowledge is unavailable because vertical features are not visible and the view is visually non-realistic. The unavailability of landmark recognition knowledge also makes the acquired route knowledge incomplete.

Experimental findings The effect of tilted 2D maps on the acquisition of spatial knowledge is weakly experimented with in the research field of spatial cognition, in spite of the popularity of navigation systems. The experiments on 3D virtual environments highlight the benefits of the egocentric vantage point for landmark and route knowledge (e.g., [33. 57, 60,73|). Landmark information is not visible in tilted 2D maps, so the benefits of the egocentric vantage point only apply to the acquired route knowledge due to the easily interpretable turning directions. According to experiments, the lack of visual realism in the symbolic 2D maps enhances the rapid acquisition of configuration knowledge (e.g., |69. 73|).

\subsection{Oblique aerial photo}

An oblique aerial photo (Figure 81 is a an aerial photo, which applies an oblique viewing angle with ground surface and is taken from a low altitude with high resolution. Oblique aerial photos have lately become common in web mapping applications. 


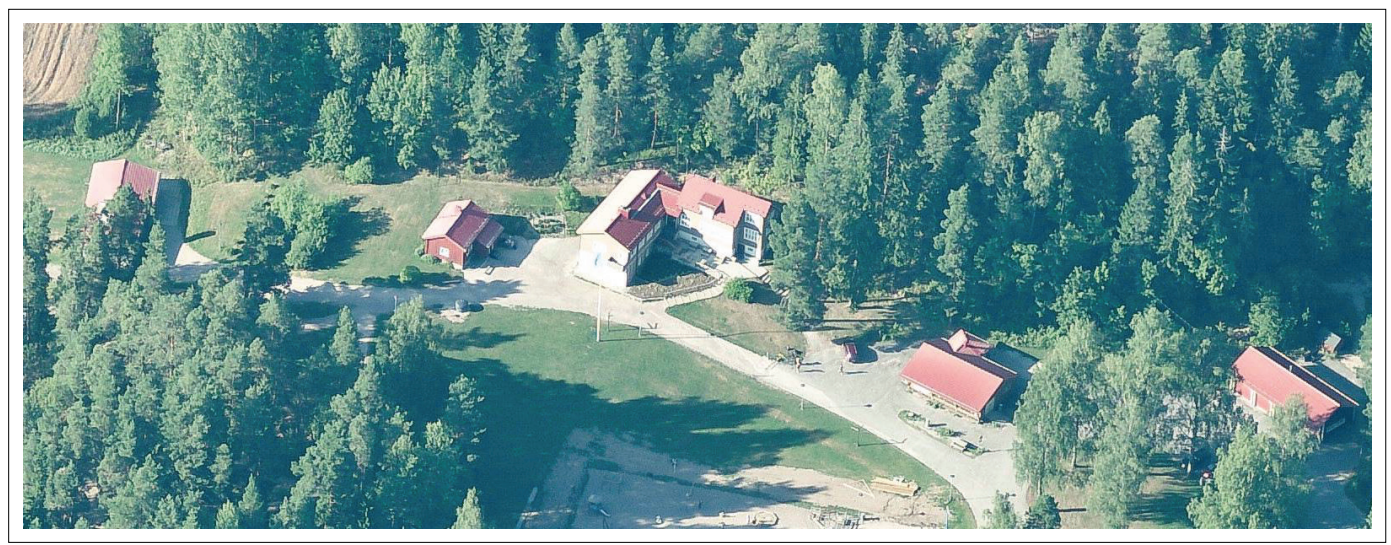

Figure 8: An example of oblique aerial photos. All rights reserved (C)BLOM [19|.

Framework-based evaluation Oblique aerial photos apply the aerial oblique vantage point, contain a high number of visible vertical features, and are photorealistic. This set of image parameters is advantageous for the acquisition of all kinds of spatial knowledge mainly due to the vantage point. Landmark details can be perceived for improved landmark recognition; landmarks can be easily linked to sequential route knowledge; and the configurational pattern of the environment is easy to observe and so refine one's cognitive map. However, in static photos, lots of features on the ground can be occluded behind tall objects. Therefore, applications with oblique aerial photos often provide the viewer with photos taken from the four cardinal directions, so that surfaces occluded from one direction can be seen from another. The photorealism in oblique aerial photos lets viewers select convenient landmark features individually based on their preferences and wayfinding styles. However, they may not perceive some small but crucial features which affect wayfinding, such as roadblocks.

Experimental findings Experiments support the expected suitability of oblique aerial photos for acquiring spatial knowledge for wayfinding. The aerial oblique vantage point is advantageous for large scale spaces, and the comprehensive coverage of visible vertical features [23| with photorealism promotes the efficient extraction of usable landmark knowledge [33|. However, controlled wayfinding experiments on oblique aerial photos have yet to be conducted.

\subsection{Oblique 3D drawing}

An oblique 3D drawing is a geospatial drawing rendered with symbolization and generalization in an oblique viewing angle (Figure 9). Drawing methods include such techniques as manual artistic drawing and computational non-photorealistic 3D rendering. For example, traditional three-dimensional touristic city maps belong to this category of geospatial images. Oblique 3D drawings are not direct visualizations of the real world, like oblique aerial photos; rather they are processed views with the aim at highlighting desired features. 
The distinction between oblique aerial photos and oblique 3D drawings is similar to that between symbolic 2D maps and aerial orthophotos.

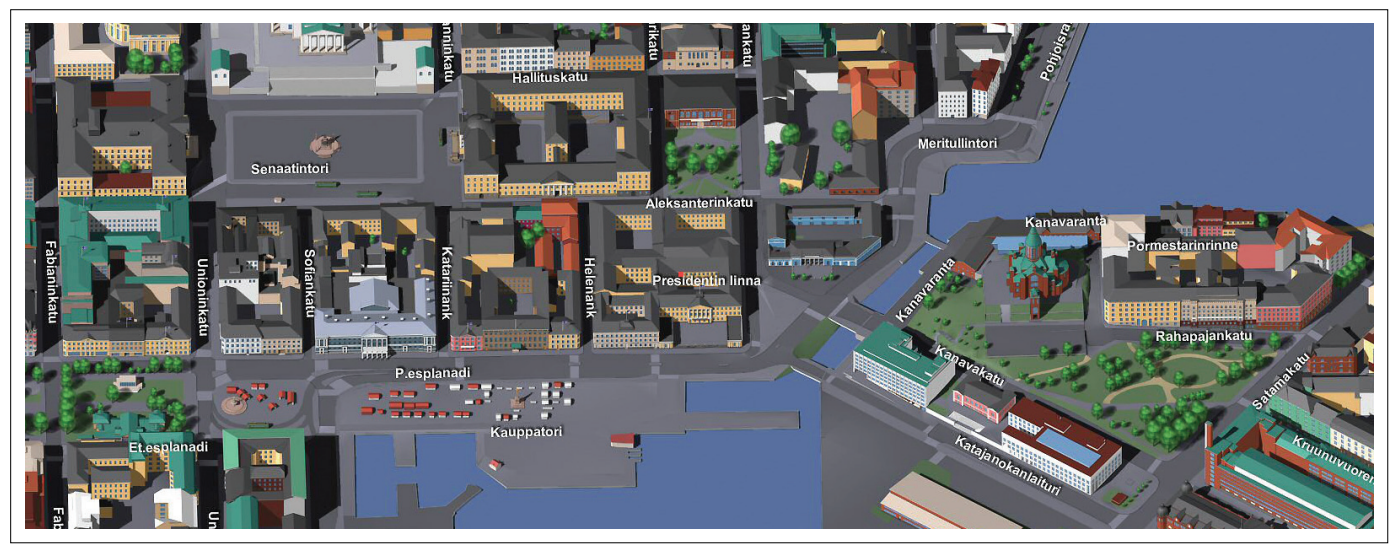

Figure 9: An example of oblique 3D drawings (C)Fontus Ltd [24|.

Framework-based evaluation Oblique 3D drawings apply the aerial oblique vantage point, contain high number of visible vertical features, and have low visual realism. The vantage point and the number of visible vertical features make it possible both to view landmark characteristics in vertical features and to recognize spatial patterns of the environment. Thus, landmark and route types of spatial knowledge as well as configuration knowledge can be acquired from oblique 3D drawings. However, the occlusion of features behind high objects is unavoidable. In applications where all feature need to be visible, a moving view is required. Low visual realism makes oblique 3D drawings selective in providing information on the drawn features. The efficiency of transferring landmark knowledge is strongly dependent on the cartographic generalization used in the drawing process. In the best case, only the meaningful characteristics of landmarks are depicted; in the worst case, the characteristics rendered provide insufficient visual information for recognizing landmarks in the real world.

An important characteristic of oblique 3D drawings is that the viewing projection can be chosen freely. For example, an oblique parallel projection may be beneficial for transferring accurate spatial knowledge because it maintains the scale throughout the view and causes lower occlusion than the perspective projection [39|.

Experimental findings Experimentation on oblique 3D drawings has found them effective for wayfinding tasks [23|. Plesa and Cartwright [61| found that viewers prefer a low-realism oblique 3D drawing to a photorealistic 3D rendering with regards to clarity, usefulness, and usability. However, experiments on visual realism in 3D virtual environments have also found that viewers prefer high realism (e.g., [12|). Further experiments are needed to investigate the ability of oblique 3D drawings for providing spatial knowledge for wayfinding. 


\subsection{Panoramic street view}

A panoramic street view (Figure 10) is a panoramic composition of photographs taken at the ground level with a wide horizontal angle of view. Panoramic street views are typically viewed in a browser, which enables moving among the photos continuously, thus creating a impression of locomotion through the real world environment. Panoramic street views are amongst the most modern types of geospatial images presented in this article, as they have recently emerged following the development of efficient geospatial data capture techniques.

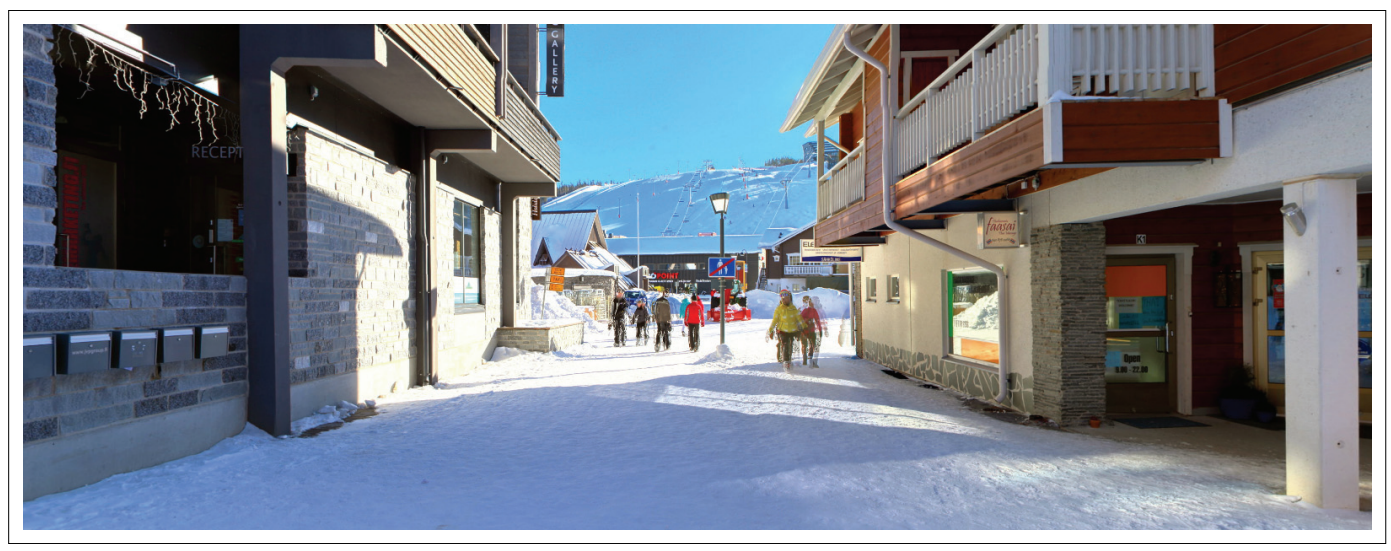

Figure 10: An example of panoramic street views (www.leviskyview.fi) (CFlycam Inc [22].

Framework-based evaluation Panoramic street views apply the ground horizontal vantage point, contain comprehensive number of visible vertical features, and are photorealistic. These parameters support the acquisition of landmark knowledge, as landmarks can be perceived just as they appear in the real world, paying attention to the details in vertical features in an egocentric frame of reference. In contrast, route knowledge and configuration knowledge can be acquired only by moving in the virtual environment, which can be inconvenient and time-consuming. Route knowledge can be collected by traversing a route through street views, which may be faster than in reality. However, acquiring configuration knowledge from panoramic street views alone requires a considerable amount of browsing.

Experimental findings Qualitative experiments verify the benefits of panoramic street views for the acquisition of landmark knowledge. Hile et al. [33| found that street photos were used at the decision points of the traversed route where the importance of landmarks is high for navigation (e.g., [36]) although aerial photos were used between the decision points. Liu et al. [43| also pointed out the usefulness of street photos at decision points as reported by their disabled users. The authors noted that users would have preferred to see more photos in advance in order to strengthen their route knowledge before proceeding on the route. Experiments are still to be done on the use of panoramic street view browsers and their ability to transfer spatial knowledge for wayfinding. It can be assumed that the acquisition proceeds in a similar way to the locomotion in real world. 


\subsection{Ground-level 3D rendering}

A ground-level 3D rendering (Figure 11) is a geospatial image based on a 3D model and rendered computationally in the egocentric frame of reference. Ground-level 3D renderings aim to model real world environments photorealistically. Physical and visual surface characteristics are draped on complex geometric models of the spatial features. In order to reach a high degree of realism, ground-level 3D renderings often apply advanced lighting models with shadows and reflections, and animated objects such as swaying trees. The complete 3D model lets the user move freely in an environment with a close visual resemblance to reality. Ground-level 3D renderings are commonly seen in virtual reality environments.

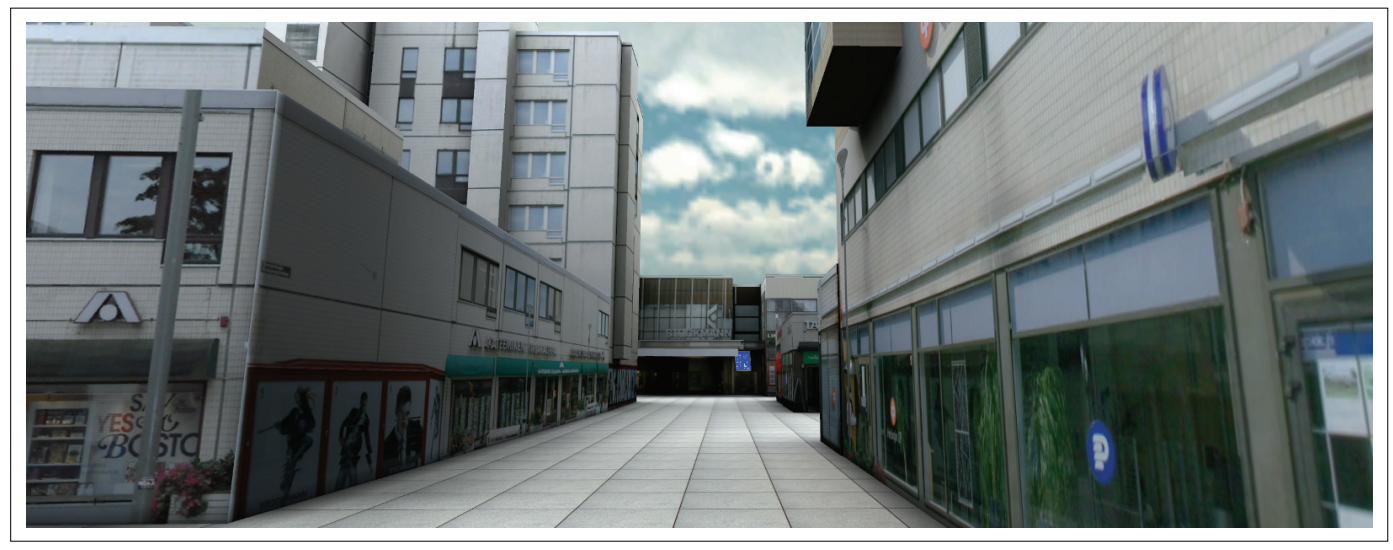

Figure 11: An example of ground-level 3D renderings (3D Tapiola) [21|.

Framework-based evaluation Ground-level 3D renderings apply the ground horizontal vantage point, contain comprehensive coverage of visible vertical features, and have a high level of visual realism. Ground-level 3D renderings support spatial knowledge for wayfinding in a similar way to panoramic street views, meaning that landmark knowledge is best acquired and route and configuration knowledge build up only with effort. However, despite the objective of photorealism, repeating textures together with the shortcomings of the underlying 3D models can make ground-level 3D renderings somewhat abstract and require additional interpretation from the viewer. Ground-level 3D renderings are usually used in virtual environments in which movement is free and continuous, giving the viewer the possibility of reaching an unlimited number of viewpoints and seeing features from all directions. However, controlling movement in a 3D virtual environment is a cognitively demanding task [57.66.73], which reduces the value of ground-level 3D renderings with regards to the acquisition of spatial knowledge.

Experimental findings Experiments have shown that ground-level 3D renderings are at their best for providing landmark knowledge for wayfinding. Oulasvirta et al. [57| reported that users applied the ground-level viewpoint of their 3D virtual environment when in need of recognizing landmark features. They also reported that the users' main problem with ground-level 3D renderings was the difficulty of controlling movement in the environment, which has also been noted in other experiments (e.g., |66.73|). Consequently, 
ground-level 3D renderings are less advantageous for acquiring route and configuration knowledge [62].

\section{Summary and conclusions}

Everyday human wayfinding is dependent on the spatial knowledge stored in internal spatial representations called cognitive maps [70] or cognitive collages [72]. In familiar environments, wayfinding is mostly an automatic cognitive process. Unfamiliar environments, such as when traveling to a novel destination, call for conscious thinking to fulfill the actual goal of wayfinding [2]. Spatial knowledge in an unfamiliar environment can be acquired through direct experience. This process is cognitively demanding and may last for years before inclusive knowledge is formed [65|. The process involves the acquisition of landmark, route, and configuration types of spatial knowledge [52 65].

Geospatial images are efficient media for acquiring spatial knowledge for wayfinding, especially in unfamiliar environments. Geospatial images have a long tradition in human history, beginning with map drawings thousands of years ago. Today, due to the development of geodata capture and visualization techniques over recent decades, various kinds of geospatial images are available to support everyday wayfinding. The everyday geospatial images are represented on planar surfaces, such as paper or a screen, which cannot capture all the detail of the real world. Different kinds of geospatial images support the acquisition of spatial knowledge for wayfinding differently. For example, traditional two-dimensional maps in particular provide configuration knowledge efficiently, whereas street photos primarily support landmark knowledge.

In the present article, we reviewed research from the disciplines of psychology and geographic information science to better understand the interconnections between geospatial images and spatial knowledge acquisition. This research provides valuable information on how geospatial images should be designed and used, but this information has not yet been widely applied. In order to narrow the gap between the two disciplines, we presented a framework which can be used to evaluate the support of geospatial images for acquiring spatial knowledge for wayfinding. The framework consists of three image parameters, each of which constructs the potential of a geospatial image for spatial knowledge transfer: vantage point, number of visible vertical features, and visual realism. Each of the parameters has different implications for the spatial informativeness of a geospatial image, but they also have important joint effects. For example, vantage point and the number of visible vertical features measure separate qualities of a view, but they are technically interconnected through the vertical viewing angle and through the issue of whether or not vertical information is available in the geodata. As another example, the specific combination of the high number of visible vertical features and high visual realism is advantageous for the acquisition of landmark knowledge.

The created evaluation framework considers large-scale, pedestrian wayfinding spaces in which the area of interest cannot, in reality, be apprehended from a single viewpoint |41|. Instead, the area can be depicted in a single, static geospatial image, such as an oblique aerial photograph. The movement of views is not considered in the framework; rather, our focus is on the characteristics of static views. However, many geospatial images, especially ones which apply the ground horizontal vantage point, are designed to be used in interactive environments where multiple views can be perceived sequentially. Therefore,

www.josis.org 
the created framework could be extended to include the movement of the view and to consider the corresponding cognitive factors, such as optic flow [62 | and perception of distance through movement [10|.

With the help of the created framework, we evaluated seven common types of geospatial images, many of which have been available with significant geographical coverage for less than a decade. For each type of geospatial image, we introduced both frameworkbased evaluations of its capability to provide spatial knowledge and experimental evidence from previous research (Section 51. For the framework-based evaluations, we specified the expected effects that each image parameter has on the acquisition of spatial knowledge from that image type. Geospatial images with aerial oblique vantage points seem to be the most beneficial for acquiring spatial knowledge for wayfinding. They represent landmark information, upon which we can build the other types of knowledge. They also represent the spatial configuration of space, which makes it possible to form internal spatial representations on the routes and configurations of the environment (Figure 4). The experimental evidence supports this expectation $[23,64 \mid$. However, we found that only symbolic 2D maps and ground-level 3D renderings have enough experimental evidence today to support strong conclusions about their capabilities for providing spatial knowledge for wayfinding. The other five types of geospatial images need more experimental attention in the future so that their cognitive characteristics can be better understood.

With regard single types of spatial knowledge, we found that panoramic street views and ground-level 3D renderings provide the best support for the acquisition of landmark knowledge because they provide detailed views of landmarks in the egocentric frame of reference (Figure 4). These image types can also provide accurate route knowledge when multiple views are observed in a browser, but this can be time-consuming. The oblique aerial photo seems to be the most beneficial image type for route knowledge, due to the simultaneous visibility of detailed landmark features and route segments. Configuration knowledge is most efficiently acquired from symbolic 2D maps and from appropriately generalized oblique 3D drawings because they provide a quick view on the configuration of important landmark features.

To conclude, previous research has found that spatial knowledge for wayfinding is based on landmark knowledge. The visibility of landmarks is central to making a geospatial image which can support wayfinding activities. Various types of geospatial images are available that support the acquisition of spatial knowledge for wayfinding differently, but insufficient experimental results exist to provide clear information about the different characteristics of the image types. Thus, more investigation is needed for the cognitive analysis of geospatial images. Further experiments are also needed in order to be able to create new geospatial images that can better support human spatial cognition. The framework presented here could be expanded to include moving views as well as various environmental and activity-related conditions, which affect wayfinding processes in the human mind.

\section{Acknowledgments}

This survey is part of an ongoing research project "Ubiquitous Spatial Communication" (UbiMap). The UbiMap project is funded by the Academy of Finland, Motive program, and is carried out in co-operation with the Finnish Geodetic Institute, Department of Geoinformatics and Cartography, and the University of Helsinki, Cognitive Science. 


\section{References}

[1] Aguirre, G. K., Detre, J. A., Alsop, D. C., And D'Esposito, M. The parahippocampus subserves topographical learning in man. Cerebral Cortex 6, 6 (1996), 823829. doi:10.1093/ cercor/6.6.823.

[2] Allen, G. L. Spatial abilities, cognitive maps, and wayfinding: Bases for individual differences in spatial cognition and behavior. In Wayfinding behavior. Cognitive mapping and other spatial processes, R. G. Golledge, Ed. The John Hopkins University Press, 1999, pp. 46-98.

[3] Allen, G. L. Preface: Routes of human spatial memory research. In Human Spatial Memory, G. L. Allen, Ed. Lawrence Erlbaum, Mahwah, NJ, 2004, pp. xiii-xx.

[4] Avraamides, M. N., AND Kelly, J. W. Multiple systems of spatial memory: Evidence from described scenes. Journal of Experimental Psychology. Learning, Memory \& Cognition 36, 3 (2010), 635-645. doi:10.1037/ a0017040

[5] Berendt, B., Barkowsky, T., Freksa, C., And Kelter, S. Spatial representation with aspect maps. In Spatial Cognition, C. Freksa, C. Habel, and K. Wender, Eds., vol. 1404 of Lecture Notes in Computer Science. Springer, Berlin, 1998, pp. 313-336. doi:10.1007/3-540-69342-4_15

[6] BLADES, M. Wayfinding theory and research: The need for a new approach. In Cognitive and Linguistic Aspects of Geographic Space. Kluwer Academic Publishers, Dordrecht, The Netherlands, 1991, pp. 137-165. doi:10.1007/978-94-011-2606-9_10

[7] Bleisch, S., AND NebiKer, S. More than rendering-printed tourist maps and an interactive 3D information system from highly detailed 3D landscape models. In Proc. XXIII International Cartographic Conference (Moscow, Russia, August 4-10 2007). Theme 17, CDROM.

[8] Brogni, A., Vinayagamoorthy, V., Steed, A., and Slater, M. Variations in physiological responses of participants during different stages of an immersive virtual environment experiment. In Proc. ACM symposium on Virtual Reality Software and Technology (VRST'06) (New York, NY, 2006), ACM, pp. 376-382. doi:10.1145/1180495.1180572.

[9] BRUnYÉ, T. T., AND TAYLOR, H. A. When goals constrain: Eye movements and memory for goal-oriented map study. Applied Cognitive Psychology 23, 6 (2008), 772 787. doi:10.1002/acp.1508

[10] BUCHNER, A., AND JANSEN-OSMANN, P. Is route learning more than serial learning? Spatial Cognition \& Computation 8, 4 (2008), 289-305. doi:10.1080/13875860802047201.

[11] BuRgess, N. Spatial memory: How egocentric and allocentric combine. Trends in Cognitive Sciences 10, 12 (2006), 551-557. doi:10.1016/j.tics.2006.10.005

[12] CARTwRIGHT, W. Landmarks and the perception of a space in web-delivered 3Dworlds. In Location Based Services and TeleCartography, G. Gartner, W. Cartwright, and M. P. Peterson, Eds., Lecture Notes in Geoinformation and Cartography. Springer, 2007, pp. 329-344. doi:10.1007/978-3-540-36728-4.

www.josis.org 
[13] Cheng, K., And Newcombe, N. Is there a geometric module for spatial orientation? Squaring theory and evidence. Psychonomic Bulletin \& Review 12 (2005), 1-23. doi:10.3758/BF03196346

[14] Christou, C., AND BÜLTHOFF, H. Using realistic virtual environments in the study of spatial encoding. In Spatial Cognition II, C. Freksa, C. Habel, W. Brauer, and K. Wender, Eds., vol. 1849 of Lecture Notes in Computer Science. Springer, Berlin, 2000, pp. 317332. doi:10.1007/3-540-45460-823

[15] Dalgarno, B., BennetT, S., AND HARPER, B. The importance of active exploration, optical flow, and task alignment for spatial learning in desktop 3D environments. Human-Computer Interaction 25, 1 (2010), 25-66. doi:10.1080 / 07370020903586670.

[16] Davies, C., And Peebles, D. Spaces or scenes: Map-based orientation in urban environments. Spatial Cognition \& Computation 10, 2-3 (2010), 135-156. doi:10.1080/13875861003759289

[17] Döllner, J. Non-photorealistic 3D geovisualization. In Multimedia Cartography, 2nd ed. Springer, Berlin, 2007, pp. 229-240. doi:10.1007/978-3-540-36651-5_16

[18] Downs, R. M., And STEA, D. Cognitive maps and spatial behavior: Process and products. In Image and Environment: Cognitive Mapping and Spatial Behavior, R. M. Downs and D. Stea, Eds. Aldine Press, Chicago, IL, 1973, pp. 8-26. doi:10.1002/9780470979587.ch41.

[19] Eniro, Blom ASA, And Blom-Pictometry. Kunnarlantie 112, Espoo, Finland. Eniro Kartat, 2012. Accessed 14.9.2012 at http://kartat.eniro.fi.

[20] FABRikant, S. I., AND Boughman, A. Communicating data quality through realism. In Proc. GIScience 2006 (Extended Abstracts) (Münster, Germany, 2006), pp. 59-60.

[21] Finnish Geodetic Institute. 3D Tapiola, 2012. Accessed 16.3.2012 at http://play. google.com/store / apps/details?id=com.FGI.Tapiola3D.

[22] Flycam Inc. Keskuskuja 3, Levi, Kittilä, Finland. Levi Skyview, 2012. Accessed 14.3.2012 at http://www.leviskyview.fi.

[23] Fontaine, S. Spatial cognition and the processing of verticality in underground environments. In Conference on Spatial Information Theory (COSIT), D. R. Montello, Ed., vol. 2205 of Lecture Notes in Computer Science. Springer, Berlin, 2001, pp. 387-399. doi:10.1007/3-540-45424-126

[24] Fontus LTD. Virtual Helsinki, 2007. Accessed 18.1.2012 at http://www. virtualhelsinki.fi.

[25] GARMin LTD. nüvi 5000, 2012. Accessed 13.2.2012 at http://www8.garmin.com/ HiRes / automotive/ nuvi5000/cf-high.jpg.

[26] GiLlner, S., AND MALLOT, H. These maps are made for walking-task hierarchy of spatial cognition. In Robotics and Cognitive Approaches to Spatial Mapping, M. Jefferies and W.-K. Yeap, Eds., vol. 38 of Springer Tracts in Advanced Robotics. Springer, Berlin, 2008, pp. 181-201. doi:10.1007/978-3-540-75388-9_11. 
[27] GolledGe, R. G. Human wayfinding and cognitive maps. In Wayfinding behavior. Cognitive mapping and other spatial processes, R. G. Golledge, Ed. The John Hopkins University Press, 1999, pp. 5-45.

[28] Google, Digital Earth Technology, DigitalGlobe, GeoEye, The GeoInforMATION GROUP, AND ZENRIN. Ariake, Tokyo, Japan. Google Maps, 2012. Accessed 12.9.2012 at http://maps.google.com.

[29] Google, And Institut CARtogràfic de CAtalunya. Barceloneta, Barcelona, Spain. Google Earth, 2010. Accessed 12.9.2012 at http://maps.google.com.

[30] Haynes, K., Barclay, J., AND PidgeON, N. Volcanic hazard communication using maps: an evaluation of their effectiveness. Bulletin of Volcanology 70 (2007), 123-138. doi:10.1007/ s00445-007-0124-7

[31] Hegarty, M., Smallman, H. S., And Stull, A. T. Choosing and using geospatial displays: Effects of design on performance and metacognition. Journal of Experimental Psychology: Applied 18, 1 (2012), 1-17. doi:10.1037 / a0026625

[32] Hegarty, M., Smallman, H. S., Stull, A. T., and Canham, M. S. Naïve cartography: How intuitions about display configuration can hurt performance. Cartographica 44, 3 (2009), 171-186. doi:10.3138/carto.44.3.171.

[33] Hile, H., Vedantham, R., Cuellar, G., Liu, A., Gelfand, N., Grzeszczuk, R., AND BORRIELLO, G. Landmark-based pedestrian navigation from collections of geotagged photos. In Proc. 7th International Conference on Mobile and Ubiquitous Multimedia (New York, NY, 2008), ACM, pp. 145-152. doi:10.1145/1543137.1543167

[34] Hirtle, S., TimpF, S., AND TENBRINK, T. The effect of activity on relevance and granularity for navigation. In Conference on Spatial Information Theory (COSIT), M. Egenhofer, N. Giudice, R. Moratz, and M. Worboys, Eds., vol. 6899 of Lecture Notes in Computer Science. Springer, Berlin, 2011, pp. 73-89. doi:10.1007/978-3-642-23196-4_5

[35] Ishikawa, T., AND MONTEllo, D. R. Spatial knowledge acquisition from direct experience in the environment: Individual differences in the development of metric knowledge and the integration of separately learned places. Cognitive Psychology 52, 2 (2006), 93-129. doi:10.1016/j.cogpsych.2005.08.003

[36] JANZEN, G., AND VAN TURENNOUt, M. Selective neural representation of objects relevant for navigation. Nature Neuroscience 7, 6 (2004), 673-677. doi:10.1038/nn1257.

[37] Kelly, J., and MCNamara, T. Spatial memory and spatial orientation. In Spatial Cognition VI. Learning, Reasoning, and Talking about Space, C. Freksa, N. Newcombe, P. Gärdenfors, and S. Wölfl, Eds., vol. 5248 of Lecture Notes in Computer Science. Springer, Berlin, 2008, pp. 22-38. doi:10.1007/978-3-540-87601-4_5

[38] Kettunen, P., AND SARJAKOSKI, L. T. A context-sensitive wayfinding ontology for hiking based on an empirical study. In Extended Abstracts from the Poster Session of the Conference on Spatial Information Theory (COSIT11) (Belfast, Maine, USA, September 12-16 2011), R. Moratz and N. Giudice, Eds. 
[39] Kettunen, P., SARjakoski, T., SARjakoski, L. T., And OKSAnen, J. Cartographic portrayal of terrain in oblique parallel projection. In Proc. 24th International Cartographic Conference (November 15-21 2009).

[40] Klatzky, R., Loomis, J., Beall, A., Chance, S., And Golledge, R. Spatial updating of self-position and orientation during real, imagined, and virtual locomotion. Psychological science 9, 4 (1998), 293-298. doi:10.1111/1467-9280.00058.

[41] Kuipers, B. Modeling spatial knowledge. Cognitive Science 2, 2 (1978), 129-153. doi:10.1207/s15516709cog02023

[42] Levine, M., Marchon, I., AND Hanley, G. The placement and misplacement of you-are-here maps. Environment and Behavior 16, 2 (1984), 139-157. doi:10.1177/0013916584162001

[43] Liu, A. L., Hile, H., Borriello, G., Brown, P. A., Harniss, M., Kautz, H., AND JOHNSON, K. Customizing directions in an automated wayfinding system for individuals with cognitive impairment. In Proc. 11th International ACM SIGACCESS Conference on Computers and Accessibility (New York, NY, USA, 2009), ACM, pp. 27-34. doi:10.1145/1639642.1639649.

[44] Lobben, A., LAWrence, M., AND Olson, J. M. fMRI and human subjects research in cartography. Cartographica 44, 3 (2009), 159-169. doi:10.3138/ carto.44.3.159.

[45] LoBben, A. K. Tasks, strategies, and cognitive processes associated with navigational map reading: A review perspective. The Professional Geographer 56, 2 (2004), 270-281. doi:10.1111/j.0033-0124.2004.05602010.x

[46] LYNCH, K. The Image of the City. MIT Press, Cambridge, MA, 1960.

[47] MacEachren, A. M. How Maps Work. Representation, Visualization, and Design. The Guilford Press, New York, NY, 1995.

[48] MacEachren, A. M., AND Ganter, J. H. A pattern identification approach to cartographic visualization. Cartographica 27, 2 (1990), 64-81. doi:10.3138/ M226-1337-2387-3007

[49] Maguire, E. A., Burgess, N., Donnett, J. G., Frackowiak, R. S., Frith, C. D., AND O'KEEFE, J. Knowing where and getting there: A human navigation network. Science 280, 5365 (1998), 921-924. doi:10.1126/ science.280.5365.921

[50] Meijer, F., Geudeke, B. L., And Van den Broek, E. L. Navigating through virtual environments: Visual realism improves spatial cognition. CyberPsychology \& Behavior 12, 5 (2009), 517-521. doi:10.1089/cpb.2009.0053.

[51] Meilinger, T., And Vosgerau, G. Putting egocentric and allocentric into perspective. In Spatial Cognition VII, C. Hölscher, T. Shipley, M. Olivetti Belardinelli, J. o. Bateman, and N. Newcombe, Eds., vol. 6222 of Lecture Notes in Computer Science. Springer, Berlin, 2010, pp. 207-221. doi:10.1007/978-3-642-14749-4_19. 
[52] Montello, D. R. A new framework for understanding the acquisition of spatial knowledge in large-scale environments. In Spatial and temporal reasoning in geographic information systems, M. J. Egenhofer and R. G. Golledge, Eds. Oxford University Press, Oxford, UK, 1998, pp. 143-154.

[53] Montello, D. R. Navigation. In The Cambridge Handbook of Visuospatial Thinking, P. Shah and A. Miyake, Eds. Cambridge University Press, NY, 2005, pp. 257-294. doi:10.2277/0521001730

[54] MÜnZer, S., Zimmer, H. D., AND BAUS, J. Navigation assistance: A trade-off between wayfinding support and configural learning support. Journal of Experimental Psychology: Applied 18, 1 (2012), 18-37. doi:10.1037/a0026553

[55] NiKOLIC, D. Evaluating relative impact of virtual reality components detail and realism on spatial comprehension and presence. Master's thesis, The Pennsylvania State University, Department of Architectural Engineering, 2007.

[56] OKsanen, J., Schwarzbach, F., Sarjakoski, L. T., And SARJakoski, T. Map design for a multi-publishing framework - case MenoMaps in Nuuksio national park. The Cartographic Journal 48, 2 (2011), 116-123. doi:10.1179/1743277411Y.0000000007

[57] Oulasvirta, A., Estlander, S., And Nurminen, A. Embodied interaction with a 3D versus 2D mobile map. Personal Ubiquitous Computing 13, 4 (2009), 303-320. doi:10.1007/ s00779-008-0209-0

[58] PASSINI, R. Spatial representations: A wayfinding perspective. Journal of Environmental Psychology 4, 2 (1984), 153-164. doi:10.1016/S0272-4944(84)80031-6.

[59] Pick, H. L., Heinrichs, M. R., Montello, D. R., Smith, K., Sullivan, C. N., AND THOMPSON, W. B. Topographic map reading. In Local applications of the ecological approach to human-machine systems, P. A. Hancock, J. Flach, J. Caird, and K. Vicente, Eds. Lawrence Erlbaum, Hillsdale, NJ, 1995, pp. 255-284.

[60] Piovesana, M., Chen, Y.-J., Yu, N.-H., Wu, H.-T., Chan, L.-W., And Hung, Y.P. Multi-display map touring with tangible widget. In Proc. International Conference on Multimedia (New York, NY, 2010), ACM, pp. 679-682. doi:10.1145/1873951.1874050

[61] Plesa, M. A., AND Cartwright, W. Evaluating the effectiveness of non-realistic 3D maps for navigation with mobile devices. In Map-based Mobile Services, L. Meng, A. Zipf, S. Winter, W. Cartwright, G. Gartner, L. Meng, and M. P. Peterson, Eds., Lecture Notes in Geoinformation and Cartography. Springer, Berlin, 2008, pp. 80-104. doi:10.1007/978-3-540-37110-6.5.

[62] Richardson, A., Montello, D., And Hegarty, M. Spatial knowledge acquisition from maps and from navigation in real and virtual environments. Memory $\mathcal{E}$ Cognition 27 (1999), 741-750. doi:10.3758/BF03211566.

[63] Sarjakoski, L. T., Kettunen, P., Flink, H.-M., LaAkso, M., RÖnneberG, M., AND SARJAKOSKI, T. Analysis of verbal route descriptions and landmarks for hiking. Personal and Ubiquitous Computing (2011). doi:10.1007/ s00779-011-0460-7. 
[64] SCHUChARDT, P., AND BOWMAN, D. A. The benefits of immersion for spatial understanding of complex underground cave systems. In Proc. 2007 ACM symposium on Virtual Reality Software and Technology (New York, NY, 2007), ACM, pp. 121-124. doi:10.1145/1315184.1315205.

[65] Siegel, A. W., AND White, S. H. The development of spatial representations of large-scale environments. In Advances in Child Development and Behavior, H. W. Reese, Ed., vol. 10. JAI, 1975, pp. 9-55. doi:10.1016/S0065-2407(08)60007-5

[66] SJÖlinder, M., HÖÖK, K., Nilsson, L.-G., And Andersson, G. Age differences and the acquisition of spatial knowledge in a three-dimensional environment: Evaluating the use of an overview map as a navigation aid. International Journal of HumanComputer Studies 63, 6 (2005), 537-564. doi:10.1016/j.ijhcs.2005.04.024

[67] Slater, M., Khanna, P., Mortensen, J., And YU, I. Visual realism enhances realistic response in an immersive virtual environment. IEEE Computer Graphics and Applications 29, 3 (2009), 76-84. doi:10.1109/ MCG.2009.55.

[68] Sorrows, M. E., AND HiRTLE, S. C. The nature of landmarks for real and electronic spaces. In Conference on Spatial Information Theory (COSIT), C. Freksa and D. Mark, Eds., vol. 1661 of Lecture Notes in Computer Science. Springer, Berlin, 1999, pp. 37-50. doi:10.1007/3-540-48384-5_3.

[69] Thorndyke, P. W., AND Hayes-Roth, B. Differences in spatial knowledge acquired from maps and navigation. Cognitive Psychology 14 (1982), 560-589. doi:10.1016/0010-0285(82)90019-6

[70] Tolman, E. A. Cognitive maps in rats and men. The Psychological Review 55, 4 (1948), 189-208. doi:10.1037/ h0061626

[71] Trowbridge, C. C. On fundamental methods of orientation and "imaginary maps". Science 38, 990 (1913), 888-897. doi:10.1126/ science.38.990.888

[72] TVersky, B. Cognitive maps, cognitive collages, and spatial mental models. In Conference on Spatial Information Theory (COSIT), A. U. Frank and I. Campari, Eds., vol. 716 of Lecture Notes in Computer Science. Springer, Berlin, 1993, pp. 14-24. doi:10.1007/3-540-57207-4_2.

[73] WALLER, D., HunT, E., AND KNAPP, D. The transfer of spatial knowledge in virtual environment training. Presence: Teleoperators \& Virtual Environments 7, 2 (1998), 129143. doi:10.1162/105474698565631

[74] WANG, R. F., AND SPELKE, E. S. Updating egocentric representations in human navigation. Cognition 77, 3 (2000), 215-250. doi:10.1016/S0010-0277(00)00105-0.

[75] WERNER, S., AND SCHMIDT, K. Environmental reference systems for large-scale spaces. Spatial Cognition and Computation 1 (1999), 447-473. doi:10.1023/ A:1010095831166

[76] Zanola, S., FAbrikant, S. I., AND COLTEKin, A. The effect of realism on the confidence in spatial data quality in stereoscopic 3D displays. In Proc. 24th International Cartographic Conference (November 15-21 2009). 
[77] Zimmons, P., AND PANTER, A. The influence of rendering quality on presence and task performance in a virtual environment. In Proc. IEEE Virtual Reality (2003), pp. 293294. doi:10.1109/VR.2003.1191170. 